\title{
Responses to tuberculin in the guinea-pig eye as a model of cell mediated immune responses in the external eye
}

\author{
R ST C DWYER, S DAROUGAR, AND MARJORIE A MONNICKENDAM \\ From the Subdepartment of Virology, Institute of Ophthalmology, Judd Street, London WC1H 9QS
}

SUMmaRY The clinical and histological findings in an animal model of ocular cell mediated immune responses are described. These were evoked in sensitised guinea-pigs by dropping tuberculin into the conjunctival sac or injecting it under the palpebral conjunctiva. When tuberculin was dropped into the conjunctival sac, higher doses were required to evoke a strong response than when it was injected subconjunctivally. When high doses of antigen were used for challenge, a mild response was observed in unchallenged contralateral eyes. The cellular response at low doses of tuberculin consisted predominantly of mononuclear cells. At higher doses the cellular infiltrate was more pronounced, and polymorphonuclear cells tended to predominate 24 hours after challenge, while at 48 hours mononuclear cells predominated.

It has been suggested that cell mediated immune responses are important in the pathogenesis of eye diseases including trachoma, herpes simplex virus infection, chronic staphylococcal infection, and vernal keratoconjunctivitis. However, there is as yet little direct evidence to support this hypothesis. Most in-vivo studies of cell mediated immune responses have been carried out in the skin, and there are relatively few studies in other tissues. We have developed an animal model using tuberculin to study cell mediated responses in the outer eye in the absence of infection or immediate-type reactions. This is similar to the diagnostic test in humans, introduced in 1909, in which tuberculin in saline or petroleum jelly was instilled into the conjunctival sac and ocular reactions were observed.' Previous studies of the reaction to tuberculin in the guinea-pig eye have involved injection of tuberculin into the guinea-pig cornea. ${ }^{2-}$ In our model, briefly described in an earlier paper, ${ }^{5}$ guinea-pigs sensitised to tuberculin are challenged by dropping antigen on to the conjunctival sac or injecting it into the palpebral conjunctiva. We here report further studies on this model.

\section{Material and methods}

Animals. Female albino guinea-pigs of the DunkinHartley strain weighing 250 to $500 \mathrm{~g}$ were used.

Correspondence to Professor S Darougar.
Antigens. Freund's complete adjuvant was used for sensitisation (Bacto Adjuvant Complete (H27Ra), Difco Laboratories); tuberculin, mammalian purified protein derivative (PPD) of tuberculin, or human PPD, lyophilised and phenol-free, was used for challenge (Ministry of Agriculture, Fisheries and Food, Central Veterinary Laboratories, Weybridge, Surrey).

Sensitisation. Animals were sensitised with four simultaneous intradermal injections of $0.2 \mathrm{ml}$ of a $50 / 50 \mathrm{v} / \mathrm{v}$ emulsion of Freund's complete adjuvant in phosphate buffered saline. Animals were skin-tested five to 10 weeks after sensitisation, and only animals with induration of at least $18 \mathrm{~mm}$ diameter were chosen for eye challenge.

Ocular challenge. Right eyes were challenged by one of two methods: (1) mammalian PPD $(0.02 \mathrm{ml})$ was instilled into the conjunctival sac and the lids closed with gentle massage to distribute the antigen; (2) human phenol-free PPD (2 $\mu \mathrm{g}$ in $0.01 \mathrm{ml})$ was injected subconjunctivally midway in the upper palpebral conjunctiva of the right eye with a $30 \mathrm{~g}$ needle. Eyes were examined and were removed for histological examinations at various times after challenge.

Clinical examination. The methods of clinical examination of the conjunctiva and cornea were as described previously. ${ }^{6}$ Eyes were examined 4, 8, 18 , 24 , and 48 hours after challenge.

Histological examination. The methods used for 
histological examination were as described previously.

\section{Results}

\section{CLINICAL FINDINGS}

\section{Challenge with drops of $P P D$}

Sensitised and control animals with clinically normal eyes were challenged in the conjunctival sac of the right eye with drops containing $0.004,0.04,0.4,4 \cdot 0$, or $40 \mu \mathrm{g}$ of mammalian PPD. No inflammatory responses were observed at any time in the eyes of unsensitised control animals. In sensitised animals there were no inflammatory responses four and eight hours after challenge.

At 24 hours three of 10 animals challenged with $0.004 \mu \mathrm{g}$ and all 10 animals challenged with $0.04 \mu \mathrm{g}$ PPD showed slight hyperaemia of the right palpebral conjunctiva, but at 48 hours all these eyes appeared normal.

At 18 hours three out of five animals challenged with $0.4 \mu \mathrm{g}$ PPD and all animals challenged with 4.0 $\mu \mathrm{g}$ PPD showed mild hyperaemia of the right palpebral conjunctiva, and at 24 hours all animals had mild hyperaemia and oedema of the palpebral conjunctiva and mild erythema of the right lid margin. Mild hyperaemia and oedema of the bulbar conjunctiva were observed at 24 and 48 hours in the animals challenged with $4 \mu \mathrm{g}$ PPD, but there was no bulbar response in the animals challenged with $0.4 \mu \mathrm{g}$ PPD. At 72 hours all eyes appeared normal.

All animals challenged with $40 \mu \mathrm{g}$ PPD showed mild to moderate hyperaemia and oedema of the palpebral and bulbar conjunctiva, slight congestion of limbal vessels, erythema of lid margins, and mucoid discharge at 18,24, and 48.hours. At 72 hours the intensity of these clinical signs had diminished. At 72 hours five guinea-pigs were killed for histological examination and the lids of the right eyes shaved before dissection. After shaving, the eyelid skin was seen to be moderately erythematous. At five days there was only mild hyperaemia of the palpebral conjunctiva, and, after shaving, the skin of the right eyelids was seen to be slightly bruised in appearance. At seven days all eyes appeared normal, but the skin of the right eyelids still appeared slightly bruised when shaved.

The left (unchallenged) eyes of about half of the animals challenged with $0.4 \mu \mathrm{g}$ PPD or more had mild hyperaemia of the palpebral conjunctiva at 24 hours.

\section{Challenge with drops of PPD after mild trauma to the conjunctiva}

Five guinea-pigs were challenged with $4 \mu \mathrm{g}$ PPD in the conjunctival sac of the right eye immediately after the upper palpebral conjunctiva had been rubbed firmly several times with a cotton wool swab. At four hours there was mild hyperaemia of the upper palpebral conjunctiva, which had disappeared at eight hours. The clinical findings at 24 and 48 hours were the same as in eyes which had been challenged without swabbing.

\section{Secondary challenge with drops of PPD}

Five guinea-pigs which had been challenged 10 days previously were rechallenged with $4 \mu \mathrm{g} \cdot \mathrm{PD}$ in the same eye. The inflammatory responses in the 48 hours following challenge were very similar to those seen in animals given a primary challenge with $4 \mu \mathrm{g}$ PPD.

\section{Challenge with subconjunctival injection of $P P D$}

Guinea-pigs were challenged in the right eye. In unsensitised animals there was mild hyperaemia of the right upper palpebral conjunctiva at four and eight hours, while the left eyes were normal. At 24 hours all eyes were normal. When sensitised animals were challenged, there was mild hyperaemia of the upper palpebral conjunctiva at four hours, but at eight hours all eyes appeared normal. At 18, 24, and 48 hours there was moderate to severe oedema and erythema of the lid margins and purulent discharge at the site of injection. There was mild to moderate hyperaemia and severe oedema of the palpebral conjunctiva, and mild to moderate hyperaemia and oedema of the bulbar conjunctiva, with mild to moderate limbal oedema and congestion of limbal vessels and mild stromal keratitis. The left, unchallenged eyes, had mild hyperaemia and oedema of the palpebral conjunctiva, and some had mild oedema of the bulbar conjunctiva at 24 hours, but at 48 hours the left eyes appeared normal. At 24 hours some animals were killed for histological examination. When the eyelids were shaved the right eyelids showed severe oedema and erythema, while the left eyelids were normal in appearance. At 72 hours right eyelids were moderately erythematous, and at five days the skin of the lids appeared to be slightly bruised.

\section{HISTOLOGICAL FIN DINGS}

\section{Challenge with drops of $P P D$}

No significant histological changes were seen at 24 or 48 hours in any of the eyes of animals challenged with 0.004 or $0.04 \mu \mathrm{g}$ PPD. In some eyes challenged with $0.4 \mu \mathrm{g}$ PPD there were small patches of mononuclear cells infiltrating the conjunctival epithelium near the lid margins and in and under the palpebral conjunctival epithelium.

In most eyes challenged with $4 \mu \mathrm{g}$ PPD there were groups of mononuclear cells with a few polymorphonuclear cells in all parts of the palpebral conjunctiva 


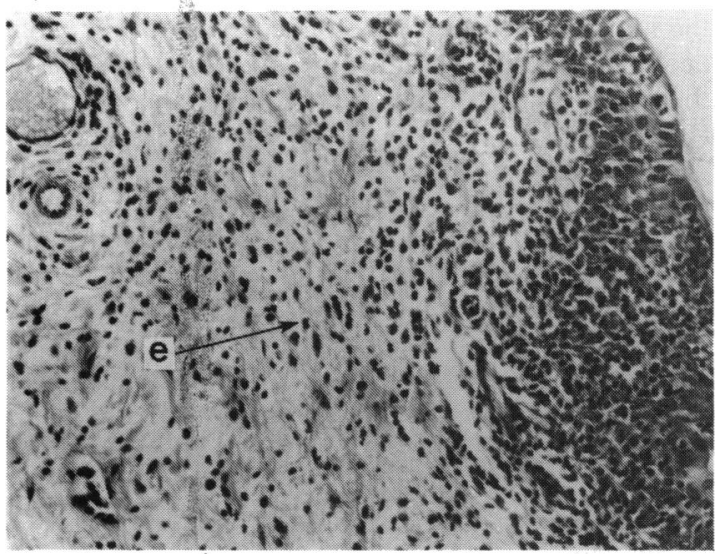

Fig. 1 Reaction at 24 hours after instillation of $40 \mu \mathrm{g} P P D$ into the conjunctival sac. Palpebral conjunctival epithelium with polymorphonuclear cells and submucosa with a predominantly mononuclear cell infiltrate and a few eosinophils (e).

and the lid margins. In some eyes there was a mild cellular infiltrate throughout the palpebral conjunctiva and the lid margins, which consisted of mononuclear cells with a few polymorphonuclear cells, and there was also a mild mononuclear cell exudate in the conjunctival sac. In the bulbar conjunctiva there were a subepithelial mononuclear cell infiltrate and some polymorphonuclear cells, mainly in the epithelium. In all eyes the limbal region had a marked cellular infiltrate, consisting of mononuclear cells with only a few polymorphonuclear cells in or just under the epithelium. At 48 hours the histological appearance was very similar to that at 24

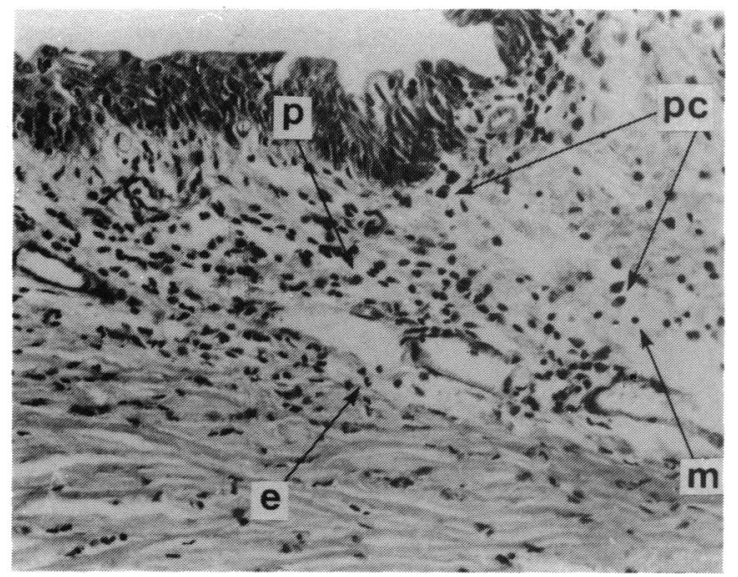

Fig. 2 Reaction at 24 hours after instillation of $40 \mu \mathrm{g} P P D$ into the conjunctival sac. Limbus with mixed cellular infiltrate consisting of mononuclear cells $(\mathrm{m})$, polymorphs (p), eosinophils (e), and plasma cells (pc). hours. Five days after challenge some eyes still had small groups of mononuclear cells in the submucosa, with a few scattered plasma cells. There were no significant changes in left, unchallenged eyes.

After challenge with $40 \mu \mathrm{g}$ PPD most eyes showed a moderate to heavy mixed cellular infiltrate, with many polymorphonuclear cells in the conjunctival epithelium, a predominantly mononuclear cell infiltrate in the submucosa, and vascular dilatation (Fig. 1). The limbus generally showed a moderate, predominantly mononuclear cell infiltrate with some polymorphonuclear cells and eosinophils and occasional plasma cells, and enlargement of limbal vessels (Fig. 2). Some left, unchallenged eyes had small patches of mononuclear cells, but most appeared normal.

\section{Challenge with subconjunctival injection of $P P D$}

At 24 hours after challenge several eyes showed a mild to moderate cellular infiltrate under the epidermis of the skin of the eyelids, in which mononuclear cells predominated. Some eyes had a heavy polymorphonuclear cell infiltrate in the epithelium of the palpebral conjunctiva close to the lid margin with consequent loss of structure. In these eyes a polymorphonuclear cell exudate was also present (Fig. 3). The submucosa showed dilated and congested vessels, and a predominantly polymorphonuclear cell infiltrate in the loose connective tissue, but not in the adjacent muscle (Fig. 3). Other eyes showed only a very few polymorphonuclear cells in the lid margin epithelium and a predominantly mononuclear cell infiltrate of the submucosa. Eosinophils were also usually present, but numbers varied considerably. The upper palpebral conjunctiva usually had a heavy

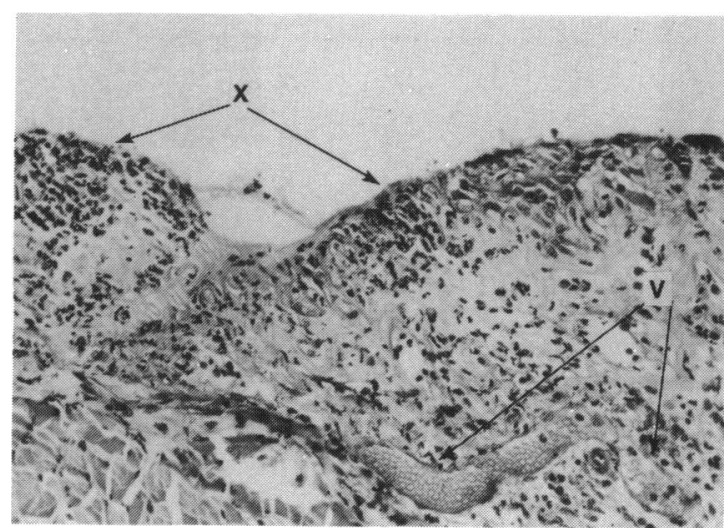

Fig. 3 Reaction at 24 hours after subconjunctival injection of 2 g PPD. Palpebral conjunctiva showing polymorphonuclear cell infiltrate with loss of structure in epithelium near lid margin; congested vessels (v), and a polymorphonuclear cell exudate $(\mathrm{x})$. 


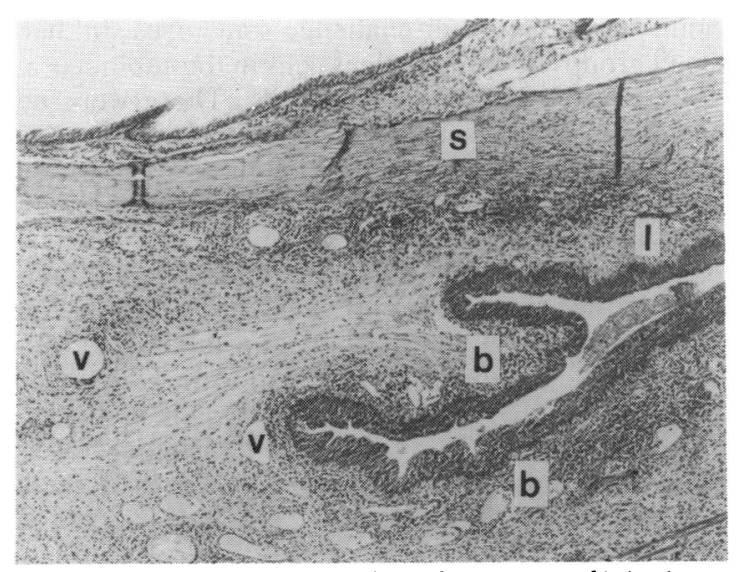

Fig. 4 Reaction at 24 hours after subconjunctival injection of $2 \mu \mathrm{g}$ PPD. Marked cellular infiltration of the bulbar conjunctiva (b), limbus (1) and sclera (s), and diluted vessels (v).

mixed cellular infiltrate under the epithelium, while the deeper tissues had a predominantly mononuclear cell infiltrate. In the lower palpebral conjunctiva most eyes had only a mild infiltrate with some polymorphonuclear cells in the epithelium, and a few mononuclear cells and eosinophils in the submucosa. In most eyes the bulbar conjunctiva had a moderate mixed cellular infiltrate in the submucosa, with a few polymorphonuclear cells in the epithelium, while a few eyes had a heavy mixed cellular infiltrate in the bulbar conjunctiva and limbus with dilated and congested vessels.

In one eye the episcleral tissue under the bulbar conjunctiva, and the underlying sclera adjacent to the limbus, the pars plana, and ciliary body all had a heavy mixed cellular infiltrate. The corneal stroma of this eye also had a heavy mixed cellular infiltrate (Figs. 4, 5).

At three, five, and seven days there was a steady diminution of the cellular infiltrate in most areas of the conjunctiva. Polymorphonuclear cells disappeared more rapidly than mononuclear cells. At seven days several eyes had small patches of cells in the palpebral conjunctiva and limbus consisting of mononuclear cells, including a few plasma cells.

Most unchallenged left eyes showed only mild mononuclear cell infiltration in the palpebral conjunctiva at 24 and 48 hours.

\section{Discussion}

We have extended our earlier studies of immune responses to PPD in the guinea-pig external eye. We have found that the maximal inflammatory response occurs 18 to 48 hours after challenge by instillation of drops or subconjunctival injection of PPD. There was a steady decline in intensity 48 hours after challenge. The timing is similar to that of guinea-pig skin reactions, which are maximal at 24 hours. ${ }^{8}$ In studies of cell mediated immune responses in the cornea the challenge has usually been carried out by injecting antigen into the cornea. ${ }^{2-49.11}$ We have shown that this traumatic procedure is unnecessary. Ocular responses can be evoked by injecting the antigen into the palpebral conjunctiva, or by dropping it into the conjunctival sac, although we found that higher doses of PPD were required to evoke a strong response when it was dropped on to the conjunctiva than when it was injected subconjunctiv-
Fig. 5 Reaction at 24 hours after subconjunctival infection of $2 \mu \mathrm{g}$ $P P D$, showing limbus (1) with a predominantly mononuclear cell infiltrate (m), and cornea (c) with a mainly polymorphonuclear cell infiltrate (p).

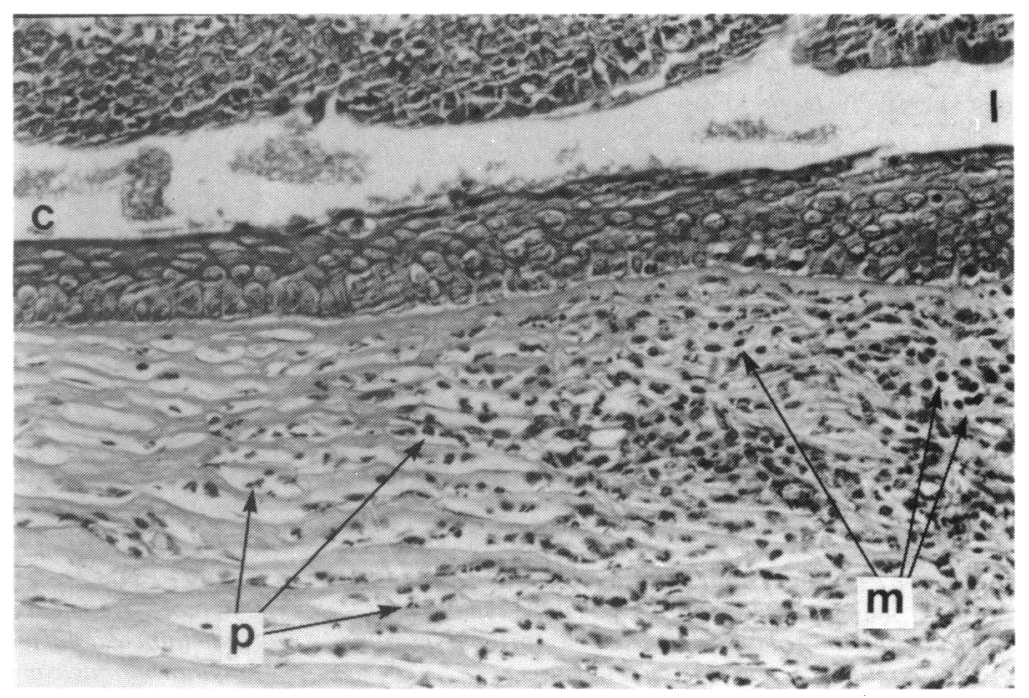


ally. The responses we observed in the palpebral and bulbar conjunctivae are similar to those reported in the limbus of animals which had been challenged by intracorneal injection, which at about 24 hours had a limbal cellular infiltrate consisting predominantly of polymorphonuclear cells. ${ }^{2+411}$ Thus we can conclude that ocular cell mediated immune responses to tuberculin in the guinea-pig eye are accompanied by a mixed polymorphonuclear and mononuclear cell response, whatever routine of challenge is used. In addition we regularly found some eosinophils, which confirms a previous report." However, there were major differences in the corneal responses which we observed compared with those of animals challenged by intracorneal injection. This is because of the damage caused by the intracorneal injection and the presence of an antigen depot in the cornea.

A heavy mixed cellular infiltrate was found in the cornea, episcleral tissue, sclera, and anterior uvea of one animal after subconjunctival injection of PPD. It was similar to naturally occurring sclerokeratitis associated with granulomatous infection, rheumatoid arthritis, and several autoimmune disorders. Silverstein and Zimmerman described a similar reaction in guinea-pigs after intravitreal injection of tuberculin, with a mixed cell infiltrate in the cornea and limbus. However, in their animals the choroid, sclera, and episclera had a predominantly mononuclear cell infiltrate.'2

A mild clinical and histological response was observed in unchallenged left eyes, particularly when higher doses of PPD were dropped into the conjunctival sac, or when it was injected. It is possible that interocular transfer of antigen may have occurred. It has been demonstrated in rabbits that subconjunctival injection of radiolabelled human serum albumin in one eye is followed by the appearance of radioactivity in the other eye. Most of this transfer does not involve the blood circulation, and it has been suggested that the major pathway is the lymphatic system. ${ }^{13}$

Our main reason for studying responses to PPD in the eye is because it is likely that cell mediated immune responses are important in ocular herpes simplex virus and chlamydial infections. However, during infection the agent is multiplying, and many immune and inflammatory responses are occurring at the same time. There are, however, some similarities between the guinea-pig ocular response to PPD and to reinfection with guinea-pig inclusion conjunctivitis (GPIC) agent (Chlamydia psittaci). In both the cellular response consists of polymorphonuclear and mononuclear cells, with a few eosinophils. We have argued that the response to reinfection with GPIC agent is predominantly cell mediated because the number of mononuclear cells present is much larger than in primary GPIC agent infection, and the number of polymorphonuclear cells is very much reduced. ${ }^{14}$ In addition, since intradermal injection of live GPIC agent evokes a cell mediated immune response in the skin, it seems reasonable to expect that live GPIC agent in the eye should also evoke an ocular cell mediated reaction. However, there are differences in the dose response curves of the eye and skin; much larger doses are needed to evoke skin responses than with eye responses. ${ }^{15}$ It is now known this is also true for skin and eye responses.

This model of ocular responses to PPD has allowed us to study the features of cell mediated immune responses in the eye. This will enable us to recognise these reactions during infection and in other conditions, when they may be present along with other immune and inflammatory reactions.

We thank Professors Barric Jones and John L Turk for advice, Mary Lang, Eileen Wilson, and Maria Pink for technical assistance, and the Medical Research Council for financial support.

\section{References}

1 Wolff-Eisner A. Ueber Tuberkulinvaseline zur Austellung der Konjunktival-reaktion, eine Modifikation der Konjunktivalprobe für dic Praxis. MMW 1909; 44: 2266-8.

2 Holley SW. Corneal reactions of normal and of tuberculous guinea-pigs to tuberculo-protein and tuberculophosphatide. Am J Pathol 1935; 11: 937-51.

3 Milner JE, Weiser RS. The corneal tuberculin reaction. Effect of systemic desensitization treatment. Arch Ophthalmol 1965; 74: $845-6$.

4 Howes EL. Cellular hypersensitivity in the cornca. An analysis of the limbus and limbal cellular infiltration by light and electron microscopy. Arch Ophthalmol 1970; 83: 475-88.

5 Dwyer R St C, Darougar S. Models of immediate and delayed hypersensitivity in the guinca-pig conjunctiva. Trans Ophthalmol Soc UK 1971; 91: 451-61.

6 Monnickendam MA, Darougar S, Treharne JD, Tilbury AM. Guinea-pig inclusion conjunctivitis as a model for the study of trachoma: clinical, microbiological, serological, and cytological studies of primary infection. BrJ Ophthalmol 1980; 64: 279-83.

7 Dwyer R St C, Darougar S, Monnickendam MA. Unusual features in the conjunctiva and cornea of the normal guinea-pig: clinical and histological studies. Br J Ophthalmol 1983; 67: 737-41.

8 Turk JL. Delayed hypersensitivity. 2nd rev. ed. Amsterdam, Oxford: North-Holland, 1975.

9 Schlossman S, Stetson CA. Vascularization of the cornea during delayed hypersensitivity reactions. J Immunol 1957; 79: 208-10.

10 Salvin SB, Gregg MB. The specificity of allergic reactions. IV. The cornea. Proc Soc Exp Biol Med 1961; 107: 478-85.

11 Friedlacnder MH, Dvorak HF. Morphology of delayed-type hypersensitivity reactions in the guinea-pig cornea. J Immunol 1977: 118: 1558-63.

12 Silverstein AM, Zimmerman LE. Immunogenic endophthalmitis produced in the guinea-pig by different pathogenetic mechanisms. Am J Ophthalmol 1959; 48: 435-46.

13 Jensen PR. Aronson SB, Pollycove M. Yamamoto E. Mechanisms of host response in the eye. III. Interocular protein transfer. Arch Ophthalmol 1967; 77: 814-7. 
14 Monnickendam MA, Darougar S, Treharne JD, Tilbury AM. Development of chronic conjunctivitis with scarring and pannus, resembling trachoma, in guinea-pigs. Br J Ophthalmol 1980; 64: 284-90.
15 Monnickendam MA, Darougar S, Tilbury AM. Ocular and dermal delayed hypersensitivity reactions in givinea-pigs following infection with guinea-pig inclusion conjunctivitis agent (Chlamydia psittaci). Clin Exp Immunol 1981; 44: 57-62.

Accepted for publication 25 June 1986. 